\title{
Un análisis de los factores que afectan la productividad de los sitios de atraque en una terminal de contenedores
}

\section{An Analysis of the Factors Affecting the Productivity of Berths at Container Terminals}

\author{
Mar-Ortiz Julio \\ Universidad Autónoma de Tamaulipas \\ Facultad de Ingeniería \\ Correo:jmar@uat.edu.mx \\ Gracia D. María \\ Universidad Autónoma de Tamaulipas \\ Facultad de Ingeniería \\ Correo:mgracia@docentes.uat.edu.mx
}

\section{Resumen}

Este artículo estudia uno de los problemas de optimización portuaria de mayor relevancia en el contexto de una terminal de contenedores: el problema de asignación de sitios de atraque. Este problema consiste en asignar los buques que llegan a una terminal de contenedores a los sitios de atraque, de manera tal que se optimice cierta función de costo. Específicamente, se describe el desarrollo de un análisis computacional del problema dinámico de asignación de sitios atraque que busca identificar el conjunto de factores operacionales y estratégicos que influyen tanto en la productividad de los sitios de atraque como en la complejidad del problema de decisión asociado. La principal motivación para desarrollar este estudio surge del supuesto de que cuando el operador de una terminal conoce cómo ciertos factores afectan un indicador de rendimiento determinado, y entiende donde radica la complejidad del problema de decisión que enfrenta, es capaz de formular mejores estrategias de operación para maximizar la competitividad de una terminal. Se propone un estudio de diseño factorial completo con el fin de apreciar la significancia de factores tales como la demanda de buques y la configuración de los arribos sobre las variables de respuesta. A partir de los resultados del análisis estadístico, el artículo concluye con la discusión de algunas implicaciones prácticas para la formulación de estrategias de operación.

Descriptores: problema de asignación de sitios atraque, logística portuaria, optimización en terminales de contenedores, cadenas de suministro, análisis estadístico.

\begin{abstract}
This paper addresses one of the main port optimization problems in the context of a container terminal: the berth allocation problem. This problem consist on assigning the arriving vessels to the berths of the terminal aiming to optimize a given cost function. Specifically, this paper describes the development of a computational study for the dynamic berth allocation problem, aiming to identify the set of operational and strategic factors that influence not only the productivity of berths, but also the computational complexity of the associated decision problem. The main motivation to develop this study arises from the assumption that when the operator of a terminal knows how certain factors affect a given performance indicator, and understand where lies the complexity of the decision problem it faces, it is able to formulate better operational strategies to maximize the competitiveness of a terminal. A full factorial design study is proposed in order to appraise the significance of factors such as demand of ships and the configuration of the arrivals on the response variables. From the statistical analysis results, this article concludes with a discussion of some practical implications for the formulation of operational strategies.
\end{abstract}

Keywords: berth allocation problem, port logistics, container terminals optimization, supply chain, statistical analysis. 


\section{INTRODUCCIÓN}

Según estimaciones de la Conferencia de las Naciones Unidas sobre Comercio y Desarrollo (UNCTAD, 2015), entre 2011 y 2014 el comercio mundial de bienes se incrementó alrededor de medio trillón de dólares, alcanzando en 2014 una cifra aproximada de US\$18.8 trillones; representando el comercio marítimo más de $80 \%$ del comercio mundial, y más de $70 \%$ en términos de valor (US\$). Los datos estadísticos de transporte en la región de América del Norte (SCT, 2015), señalan que durante ese mismo año se movieron más de 57.3 millones de TEUs (unidades equivalentes para referirse contenedores de 20 pies), constituido de la siguiente manera: Estados Unidos (81.07\%), Canadá $(9.73 \%)$ y México (9.20\%). En el contexto de América Latina y el Caribe, cuatro países explican más de $57 \%$ del tráfico de contenedores en la región, misma que durante el 2014 movió más de 45.9 millones de TEUs (figura 1). Esta cantidad constituyó $7.3 \%$ del tráfico mundial de contenedores durante ese mismo año.

Los 120 puertos considerados en el Ranking 2014 de la Comisión Económica para América Latina y el Caribe (CEPAL, 2014), presentan una gran heterogeneidad en el comportamiento de los movimientos portuarios; promediando un crecimiento de $1.3 \%$, respecto al año anterior. Se destaca el crecimiento de países como Colombia (con un alza de 7.0\%), Perú $(8.7 \%)$ y Ecuador $(7.9 \%)$. Pese al crecimiento marginal observado en el último año, la tendencia de crecimiento obliga a los operadores de las terminales de contenedores a revisar sus mecanismos de operación, con el fin de desarrollar estrategias y soluciones más eficientes para las operaciones de carga, descarga y transbordo de contenedores, que permitan hacer frente a las nuevas tendencias del mercado y de las líneas navieras que apuntan hacia un incremento en el tamaño de los buques y una disminución en la frecuencia de los arribos a las terminales portuarias.

En este contexto, el uso de herramientas cuantitativas para la optimización de operaciones portuarias ha recibido un creciente interés en el mundo, por parte de practicantes e investigadores. Dentro de una terminal de contenedores se pueden encontrar una amplia variedad de problemas logísticos y de programación que pueden resolverse de forma eficiente mediante técnicas de ingeniería industrial e investigación de operaciones (Macharis y Bontekoning, 2004; Mar et al., 2016). En la literatura existe una amplia variedad de revisiones actualizadas del estado del arte (Vis y Koster, 2003; Steenken et al., 2004; Carlo et al., 2014; Bierwirth y Meisel, 2015), y libros (Günther y Kim, 2005; Kim y Günther,
2007; Böse, 2011) dedicados a temas de optimización en operaciones portuarias.

Desde una perspectiva operacional, el proceso de transferencia de contenedores dentro de una terminal se puede dividir en cuatro elementos: operaciones en muelle, operaciones en patio, operaciones dentro del área de almacenamiento y la operación en puerta. Desde una perspectiva sistémica, una terminal de contenedores puede verse como un sistema complejo que contiene varias entidades físicas (sitios de atraque, espacio de almacén, grúas, personal), financieras (costos de operación, tarifas, salarios), y condicionales de la operación (niveles de contaminación, sistemas de control e inspección, sistemas de seguridad) que interactúan con otras entidades externas como transportistas y sociedad en general, por lo que el diseño de soluciones integradas y coordinadas toma relevancia en el contexto de la optimización portuaria. Se debe entender que el determinar, por ejemplo, la mejor estrategia para la atención de buques no solo afecta a la productividad de la terminal de contenedores en términos de equipo necesario y tiempos; sino que también afecta el tiempo de estadía de los camiones en las colas afectando los tiempos de espera y a la congestión.

La gran complejidad de los problemas que enfrenta la industria portuaria lleva implícito que hasta el momento no se conozcan técnicas que resuelvan óptimamente los casos reales en tiempos de cómputo razonables. Sin embargo, los operadores de las terminales deben tomar decisiones continuamente. Es por ello que los responsables necesitan sistemas de gestión y control que les brinden soporte durante el proceso de toma de decisiones, y así definir estrategias de operación adecuadas para la operación de la terminal. Para poder desarrollar dichos sistemas de soporte a la toma de decisiones se requiere de una fundamentación teórica, la cual brindan los modelos matemáticos, quienes permiten contemplar un amplio conjunto de alternativas de forma rápida y objetiva mediante su resolución algorítmica.

Este artículo estudia uno de los problemas de optimización portuaria de mayor relevancia en el contexto de una terminal de contenedores: el Problema de Asignación de Sitios de Atraque (BAP, acrónimo en inglés de Berth Allocation Problem). Este problema consiste en asignar los buques que llegan a una terminal de contenedores a los sitios de atraque donde se atenderán, de forma tal que se optimice cierta función de costo. En este documento se describe el desarrollo de un análisis computacional del Problema Dinámico de Asignación de Sitios de Atraque (DBAP) que busca identificar el conjunto de factores operacionales y estratégicos que influyen tanto en 
la productividad de los sitios de atraque como en la complejidad del problema de optimización asociado.

La principal motivación para desarrollar este estudio surge del supuesto de que cuando el operador de una terminal conoce cómo ciertos factores operacionales y estratégicos afectan un indicador de rendimiento determinado, y entiende dónde radica la complejidad del problema de decisión que enfrenta, es capaz de formular mejores estrategias de operación que maximicen la competitividad de una terminal. Por ejemplo, para minimizar los tiempos de atención a buques, pueden surgir varias estrategias, como descargar primero y cargar después, carga y descarga simultanea en bahías individuales o mixtas, entre otras. Sin embargo, la eficiencia de cada estrategia, aún dentro de una misma terminal, depende de varios factores; por ejemplo, la cantidad de contenedores a cargar y descargar, el número de vehículos asignados a la operación, la composición de la carga (por bahía, cuantos contenedores de 20 y 40 pies), la asignación de grúas, entre otros. Estos mismos factores, pueden influir sobre la complejidad de los problemas de decisión asociados a las operaciones de carga y descarga de buques, como lo son el plan de estiba y la programación de grúas de muelle. Haciendo que, por ejemplo, la cantidad de contenedores a mover sea relevante no solo para seleccionar la mejor estrategia de operación; sino que también sea relevante para determinar qué tan complejo será encontrar una buena solución para los problemas de decisión asociados. Al entender en qué factores radica la complejidad de un problema, también es un paso importante para el diseño de algoritmos especializados de alto rendimiento. Por ejemplo, Derpich y Vera (2006) analizaron la complejidad computacional del Multidimensional Knapsack Problem, usando la planicie del poliedro, el número restricciones y el número de variables del problema, como variables explicativas del número de iteraciones de un algoritmo de ramificación y poda. A partir de los resultados del análisis, los autores describen una estrategia basada en la información que ofrece la forma del poliedro para definir prioridades en la ramificación de variables en un algoritmo de ramificación y poda.

Con base en la discusión previa, la principal contribución de este artículo consiste en identificar un conjunto de factores operacionales y estratégicos que afectan la productividad de los sitios de atraque y la complejidad de su problema de decisión asociado. El resto de este documento se organiza de la siguiente manera: primero se describe en qué consiste el $\mathrm{BAP}$, se discuten algunas variantes del mismo y se presenta la formulación para el DBAP. Posteriormente, se describe el marco experimental, discutiendo y analizando los re- sultados del estudio computacional. Finalmente se discuten algunas implicaciones prácticas para el diseño de estrategias de operación y se ofrecen las conclusiones del estudio.

\section{El PROBlema de ASIGNACIÓN DE SITIOS DE ATRAQUe}

Cuando los buques llegan a un puerto, deben esperar a que se les asigne una posición donde atracar en el muelle de la terminal destino. El muelle es una plataforma que sobresale en el agua para facilitar la carga y descarga de los contenedores. Los lugares donde se llevan a cabo estas tareas en el puerto se denominan atracaderos, o sitios de atraque. Los sitios de atraque están equipados con grúas de muelle que se utilizan para cargar y descargar contenedores; quienes se transfieren, respectivamente, desde y hacia el patio por una flota de vehículos.

El BAP consiste en asignar los buques que llegan a una terminal, a los sitios de atraque. Desde una perspectiva general, este problema involucra dos decisiones relacionadas entre sí: dónde y cuándo asignar los buques a los sitios de atraque (Cordeau et al., 2005). Típicamente, el horizonte de planificación del BAP es de una semana, y el plan de atraque se actualiza todos los días. Por lo general, el objetivo del BAP consiste en minimizar el tiempo total de servicio para todos los buques (Meisel y Bierwirth, 2009). El BAP ha sido uno de los problemas de optimización portuaria más estudiados en la literatura (Imai et al., 1997 y 2001; Lim, 1998; Nishimura et al., 2001; Park y Kim, 2002; Hansen et al., 2008; Zhen, 2015), incorporando en los últimos años otras decisiones relacionadas a la planificación de carga (Park y Kim, 2003), la programación de grúas (Meisel y Bierwirth, 2013; Frojan et al., 2015; Iris et al., 2015), y demás problemas relacionados dentro de una terminal de contenedores (Du et al., 2015; Tao y Lee, 2015), quienes en algunos casos parecen corresponder a una estructura de programación multinivel. Se refiere a Carlo et al. (2015), Bierwirth y Meisel (2010 y 2015) para una revisión actualizada las operaciones en muelle dentro de una terminal de contenedores, incluido el BAP y sus variantes.

Bajo una tipificación general, el BAP puede clasificarse de acuerdo con la distribución de los sitios de atraque en: discreto (si los sitios de atraque están fijos) y continuo (si no lo están, donde se permite al buque atracar en cualquier lugar del muelle) o bien, de acuerdo con el supuesto de llegada de los buques en estático (si se asume que todos los buques se encuentran en la terminal antes de que se inicie la planificación) y dinámico (si se asume que los buques pueden llegar a la ter- 


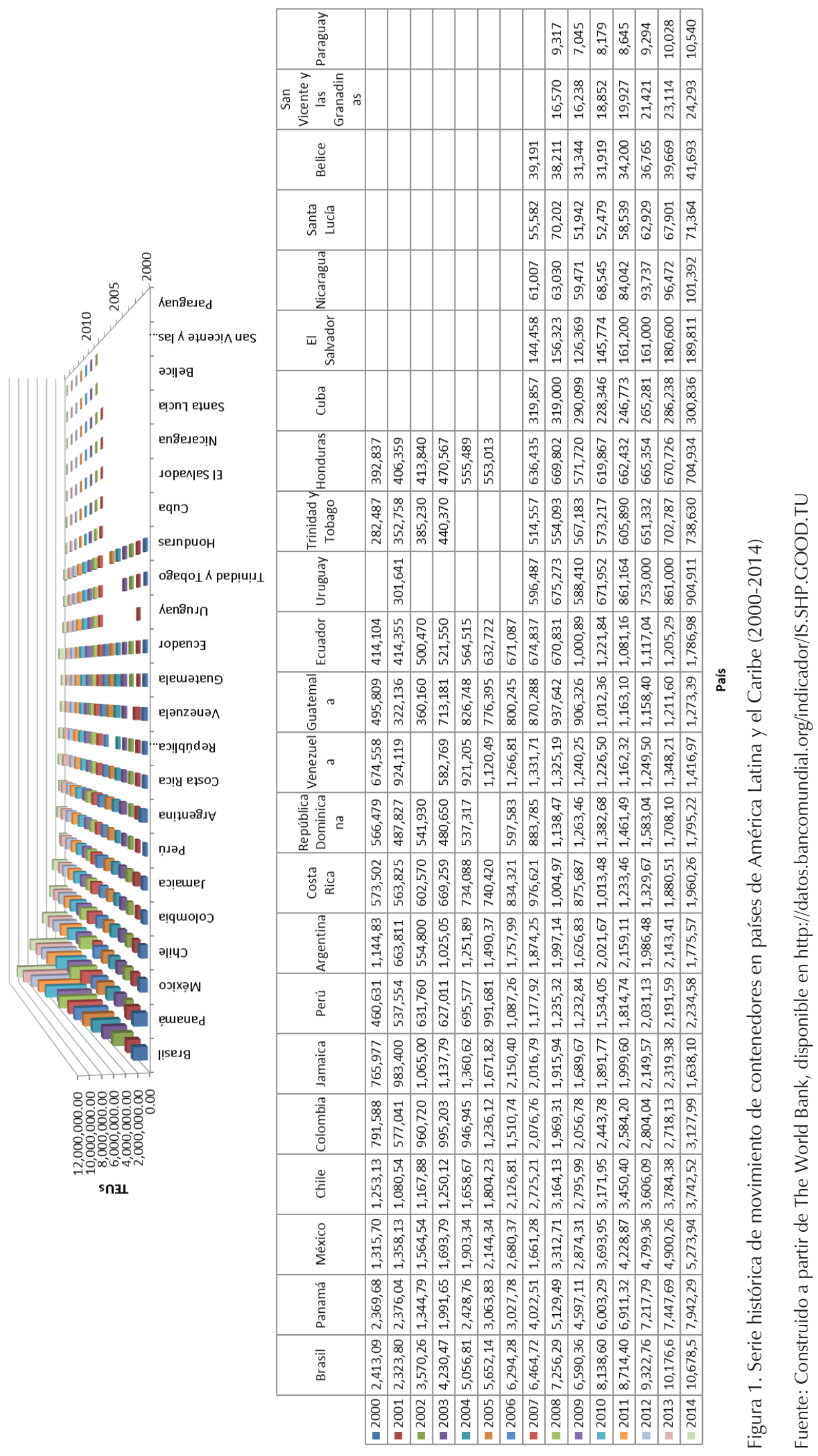


minal en algún momento dentro del horizonte de planificación) (Giallombardo et al., 2010).

En el caso discreto el BAP se puede modelar como un problema de programación de máquinas en paralelo (Imai et al., 1997), donde se trata a cada buque como un trabajo y a cada muelle como una máquina, en donde el tiempo de llegada del buque al muelle es el tiempo de liberación del trabajo. En el caso continuo, el BAP es un problema de corte en dos dimensiones con restricciones adicionales ( $\mathrm{Hu}, 2015)$; por lo que en ambos casos, el BAP es un problema NP-difícil (Garey y Johnson, 1979). El caso estático del BAP se propuso por (Imai et al., 1997) quienes probaron que se reduce a un problema de asignación bidimensional que puede resolverse fácilmente por el método Húngaro. El caso dinámico, es similar al caso estático, pero con la diferencia de que los buques pueden llegar mientras el trabajo de los sitios de atraque está en proceso (Nishimura et al., 2001). El BAP dinámico se reduce a un problema de asignación en tridimensional con algunas restricciones adicionales (Imai et al., 2001).

Esta investigación se enfoca específicamente al caso discreto-dinámico de asignación de sitios atraque. Este es un problema NP-difícil aún con un solo sitio de atraque, que se reduce a un problema de minimizar el tiempo total de terminación de tareas con fechas de liberación en una sola máquina, por lo que la regla de primero en llegar - primero en ser atendido (la cual puede ser adoptada por razones de imparcialidad) no resulta ser óptima (Hansen y Oguz, 2003).

Las decisiones de asignación de sitios de atraque tienen inferencia directa en los costos de operación de una terminal y los tiempos de atención a buques; sin embargo, en la literatura revisada no se encontraron estudios enfocados a identificar el conjunto de factores operacionales y estratégicos que afectan la productividad de los sitios de atraque y la complejidad de su problema de decisión asociado, que permitan a los operadores de una terminal formular estrategias de operación eficientes que maximicen su competitividad. Por lo que esta investigación cierra el vacio identificado.

\section{FORMULACIÓN}

El problema de asignación de sitios de atraque consiste en determinar la mejor manera de asignar cada uno de los buques que llegan a una terminal de contenedores a un sitio de atraque, para operaciones de carga y descarga, de forma tal que se optimice alguna función de costo. Las características de los buques, de la terminal y de los recursos de la misma, determinan qué restricciones deben cumplirse para obtener una solución factible.
Además, pueden considerarse diferentes funciones de costo para medir la eficiencia de la terminal; aunque el tiempo total que el buque pasa en la terminal (hora de salida del buque menos su hora de llegada) continua siendo un criterio importante para evaluar la eficiencia de un puerto.

En el caso discreto-dinámico, se asume que la terminal portuaria cuenta con un número fijo de sitios de atraque que le permiten dar servicio a varios buques al mismo tiempo; pero cada sitio de atraque es capaz de atender un solo buque a la vez o permanecer ocioso por un tiempo. No existe restricción en cuanto a qué buques se pueden atender por cada sitio de atraque; por lo que puede atender cualquier sitio de atraque a cualquier buque. Sin embargo, el tiempo de servicio en cada sitio de atraque es dependiente tanto del buque como del sitio de atraque; en este sentido, un buque en un determinado sitio de atraque puede tomar hasta 15\% más del tiempo que en otro sitio de atraque diferente (Imai et al., 2001). Se asume también que la llegada de los buques a la terminal puede ocurrir después de que los sitios de atraque hayan iniciado operación.

Sean $V=\{j=1, \ldots, T\}$ y $B=\{i=1, \ldots, I\}$, respectivamente, el conjunto de buques que llegan a una terminal de contenedores, y el conjunto de sitios de atraque disponibles en dicha terminal. De cada buque $j$ se conoce su tiempo estimado de llegada a la terminal $\left(A_{j}\right)$, así como el tiempo estimado de servicio $\left(c_{i j}\right)$ en operaciones de carga y descarga, en el sitio de atraque $i$. De cada sitio de atraque $i$ se conoce el tiempo a partir del cual está disponible para operar $\left(S_{i}\right)$. A cada sitio de atraque, (Hansen y Oguz, 2003) le asignan un orden inverso, con un índice $k$, sobre el cual los buques serán atendidos, tal que $O=\{k=1, \ldots, T\}$ es el conjunto de lugares disponibles para el servicio en un sito de atraque. A partir de lo anterior, se definen los siguientes subconjuntos

$$
\begin{aligned}
& P_{k}=\{m \in O \mid m>k\} \\
& W_{i}=\left\{j \in V \mid A_{j}>S_{i}\right\}
\end{aligned}
$$

donde (1) representa el conjunto de posiciones posteriores al orden $k$, y (2) el conjunto de todos los buques que llegan después de que el sitio de atraque $i$ está disponible. Finalmente, considerando el orden inverso en el cual los buques $j$ se atienden en el sitio de atraque $i$, se definen las siguientes variables de decisión:

$x_{i j k}=\{0,1\}: 1$ si el buque $j$ se sirve en la última $k$-ésima posición disponible en el sitio de atraque $i, 0$ en otro caso. 
$y_{i j k}=$ tiempo ocioso del sitio de atraque $i$ antes de la llegada del buque $j$, que será atendido en la última $k$-ésima posición.

La figura 2 muestra un ejemplo de una posible asignación y planificación para 4 buques y 3 sitios de atraque. En este caso, el buque 1 se coloca en el sitio de atraque 1, de forma simultánea, el buque 3 se coloca en el sitio de atraque 2 . El espacio en blanco entre el buque 1 y el buque 2, representa el tiempo de ocio del sitio de atraque 1 . Es decir, el tiempo durante el cual el sitio de atraque 1 estuvo inactivo.

\begin{tabular}{|l|l|l|l|l|}
\hline & \multicolumn{4}{|c|}{ Tiempo } \\
\hline Atraque 1 & Buque 1 & & Buque 2 & \\
\hline Atraque 2 & \multicolumn{3}{|c|}{ Buque 3 } & \\
\hline Atraque 3 & \multicolumn{4}{|c|}{} \\
\hline
\end{tabular}

Figura 2. Ejemplo de planificación de sitios de atraque

El DBAP se formula como

$\min \sum_{i \in B} \sum_{j \in V} \sum_{k \in O}\left(k c_{i j}+S_{i}-A_{j}\right) \cdot x_{i j k}+\sum_{i \in B} \sum_{j \in V} \sum_{k \in O} k \cdot y_{i j k}$

sujeto a:

$$
\begin{array}{ll}
\sum_{i \in B} \sum_{k \in O} x_{i j k}=1 & \forall j \in V \\
\sum_{j \in V} x_{i j k} \leq 1 & \forall i \in B, k \in O \\
\sum_{l \in V} \sum_{m \in P_{k}}\left(c_{i l} \cdot x_{i l m}+y_{i l m}\right)+y_{i j k} \geq\left(A_{j}-S_{i}\right) \cdot x_{i j k} & \forall i \in B, j \in W_{i}, k \in O(6) \\
x_{i j k} \in\{0,1\} & \forall i \in B, j \in V, k \in O(7) \\
y_{i j k} \geq 0 & \forall i \in B, j \in V, k \in O(8)
\end{array}
$$

La función objetivo (3) minimiza los tiempos totales de espera y trabajo en la atención de todos los buques que llegaran durante el horizonte de planeación definido, así como los tiempos de ocio de los sitios de atraque. La restricción en la ecuación (4) asegura que todos los buques sean atendidos, mientras que la restricción en la ecuación (5) establece una condición de factibilidad, evitando que dos o más buques se atiendan en un mismo sitio de atraque al mismo tiempo. La restricción en (6) computa los tiempos de ocio de los sitios de atraque, además de que condiciona la atención de los buques a un tiempo después de su llegada. Finalmente, (7) y (8) hacen referencia a las restricciones técnicas.

\section{EXPERIMENTACIÓN Y ANÁLISIS}

\section{DISEÑO EXPERIMENTAL}

Esta sección describe el marco experimental utilizado para identificar el conjunto de factores operacionales y estratégicos que influyen tanto en la productividad de los sitios de atraque, como en la complejidad del problema de decisión asociado (el DBAP previamente modelado). Determinar cómo ciertos factores operacionales y estratégicos afectan la productividad de los sitios de atraque, y la complejidad del problema de decisión asociado, permite al operador de una terminal formular mejores estrategias de operación que maximicen la competitividad de la misma. Para medir la productividad de los sitios de atraque, se utiliza como criterio directo el tiempo de ocio total $\left(\sum_{i j k} y_{i j k}\right)$ de los sitios de atraque; mientras que para medir la complejidad del DBAP, se utiliza como criterio indirecto el gap de dualidad. El gap de dualidad mide la distancia relativa entre el valor de la cota superior e inferior en el árbol de ramificación y poda.

Se propone un estudio de diseño factorial completo con el fin de apreciar la significancia de factores operacionales y estratégicos en la asignación de sitios de atraque sobre las variables de respuesta. Lo que está en cuestión aquí, es ver qué sucede con la productividad de los sitios de atraque y la complejidad del DBAP asociado cuando varían los factores. Para esto, se requieren varias instancias de diferentes tamaños para desarrollar la experimentación computacional; considerando un conjunto de factores sobre los cuales existe cierta conjetura de afectar tanto la complejidad del problema como la productividad de los sitios de atraque: configuración de los arribos (dispersión en el tiempo de llegada entre buques), demanda (número de buques que llegan a la terminal en el horizonte de planificación), capacidad de servicio de la terminal (número de sitios de atraque disponibles para dar servicio) y variabilidad del tiempo de servicio.

Un tipo de instancia (o clase) se define por una combinación de estos cuatro factores. Cabe señalar que el número de clases que se obtuvieron es $(3)(3)(2)(2)=36$. Para cada clase se generaron 10 réplicas (problemas) usando diferentes semillas aleatorias, resultando en una batería de 360 instancias. Con la finalidad de evitar cualquier desviación en el diseño de las instancias, los tiempos de llegada de los buques se generaron usando 
una distribución exponencial y los tiempos de servicio utilizando una distribución Erlang (Imai et al., 2001); el número de buques que pueden llegar por semana a una terminal, corresponde a instancias propuestas por (Giallombardo et al., 2010); finalmente, el número de sitios de atraque corresponde a instancias de tamaño moderado propuestas por (Cordeau et al., 2005). La tabla 1 muestra los factores considerados, los niveles para el análisis y los rangos de valores utilizados para la generación de los casos de prueba. En todos los casos se asume que todos los sitios de atraque están disponibles al inicio de la operación. Todos los experimentos se ejecutaron sobre una Workstation HP Z800 con procesador Intel Xeon X5647 que opera a $2.93 \mathrm{GHz}$ y pose $12.00 \mathrm{~GB}$ RAM. El tiempo de cómputo límite se fijó en 4500 segundos (tiempo suficiente para al menos encontrar una solución factible para todas las instancias), después del cual, se obtienen las estadísticas referentes al gap de dualidad, el tiempo de ocio total, el tiempo de cómputo (cuando es menor al tiempo límite), y la mejor solución encontrada hasta entonces. El modelo de optimización se resolvió usando IBM ILOG CPLEX Optimization Studio versión 12.6.1, y el análisis estadístico se efectúa con MINITAB versión 16.

\section{ANÁLISIS ESTADÍSTICO}

Tanto el gap de dualidad como el tiempo de ocio total muestran una relación de dependencia, es decir, están correlacionadas. Por este motivo, el análisis estadístico recae sobre una prueba de análisis multivariado de varian$z a$ (MANOVA). El uso del MANOVA permite probar simultáneamente si las variables de respuesta se ven alteradas por la manipulación de las variables independientes (factores), a la vez que: (i) incrementa la potencia de la prueba, al ser capaz de detectar diferencias demasiado pequeñas para detectarse a través de pruebas de Análisis de Varianza (ANOVA) individuales; (ii) detecta patrones de respuesta multivariantes; y (iii) minimiza la probabilidad de cometer el error tipo I (rechazar incorrectamente la hipótesis nula) para el conjunto total de comparaciones, al probar todas las variables de respuesta de forma simultánea. Sin embargo, el MANOVA requiere verificar nueve supuestos:

1) Las variables dependientes o respuestas deben ser continuas.

2) Las variables independientes deben constituir dos o más grupos categóricos independientes.

3) Independencia de observaciones, lo que significa que no existe relación entre las observaciones en cada grupo o entre los grupos mismos.

4) Un tamaño de muestra adecuado.

5) No hay valores atípicos univariados o multivariados.

6) Normalidad multivariante.

7) Una relación lineal entre cada par de variables dependientes para cada grupo de la variable independiente.

8) Una homogeneidad de las matrices de varianza-covarianza.

9) No multicolinealidad.

Con el fin de examinar individualmente cada una de las dos variables de respuesta y de identificar los efectos significativos para complementar el análisis del MANOVA, se revisan los resultados univariantes del ANOVA para cada variable de respuesta dentro de este experimento. Cada ANOVA evalúa los efectos de la capacidad de servicio de la terminal (F1), la demanda (F2), la variabilidad del tiempo de servicio (F3), la configuración de los arribos (F4) y su interacción a dos niveles para cada factor sobre cada variable de respuesta. Los supuestos de normalidad, homogeneidad de varianza e independencia de los residuales se verificaron para cada ANOVA, sin encontrar bases para cuestionar la validez del experimento.

La tabla 2 muestra el efecto de los factores estudiados sobre la variable dependiente gap de dualidad. El $p$-valor indica el nivel de significancia de cada factor (o combinación de los niveles de dos factores, respectivamente). Así, si el nivel de significancia de un determinado factor

Tabla 1. Factores y niveles utilizados en la generación de las instancias

\begin{tabular}{lccc}
\hline \multicolumn{1}{c}{ Factor } & \multicolumn{3}{c}{ Niveles } \\
\hline $\begin{array}{l}\text { (F1) Número de sitios de atraque } \\
\text { (F2) Número de buques }\end{array}$ & 50 & 70 & 40 \\
$\begin{array}{l}\text { (F3) Tiempo de servicio } \\
\begin{array}{l}\text { (F4) Tiempo de llegada entre } \\
\text { buques }\end{array}\end{array}$ & Homogéneos $\sim$ Erlang $(1 / 4,3)$ & Heterogéneos $\sim$ Erlang $(1 / 6,3)$ \\
\hline
\end{tabular}

Nota: las 360 instancias están disponibles en:

https://www.dropbox.com/sh/zegfOvdrnekbp2u/AAAgd9bdFfRgkG0KJZ_7ogM2a?dl=0 
está por debajo del nivel de significancia de $\alpha=0.050$, significa que se puede considerar ese factor como estadísticamente significativo a $95 \%$, y por lo tanto, tiene una influencia sobre el gap de dualidad. Los resultados del ANOVA muestran que la demanda (F2), la capacidad de servicio de la terminal (F1) y la interacción entre ambos son estadísticamente significativos para la complejidad del problema. La configuración de los arribos (F4) es apenas significativo al 0.05 . No obstante, no se pudo demostrar que la variabilidad del tiempo de servicio (F3) afecte la calidad de las soluciones obtenidas y, por lo tanto, la complejidad del problema. En su conjunto los dos primeros factores y su interacción contribuyen a explicar
$41.64 \%$ de la varianza total del gap de dualidad. El valor de $R^{2}$ ajustado $=40.86 \%$ indica una adecuación moderada del modelo. Estos resultados no son sorprendentes si consideramos que F1 y F2 son respectivamente el número de buques y el número de sitios de atraque. Los cuales están directamente relacionados al número de variables y restricciones del problema. Sin embargo, el hecho de que el tiempo de llegada entre los buques (F4) sea un factor significativo implica que la dispersión con la cual llegan los buques afecta la complejidad del problema. El análisis de los residuales (figura 3) muestra que los supuestos del ANOVA se satisfacen: la prueba de normalidad de Kolgomorov-

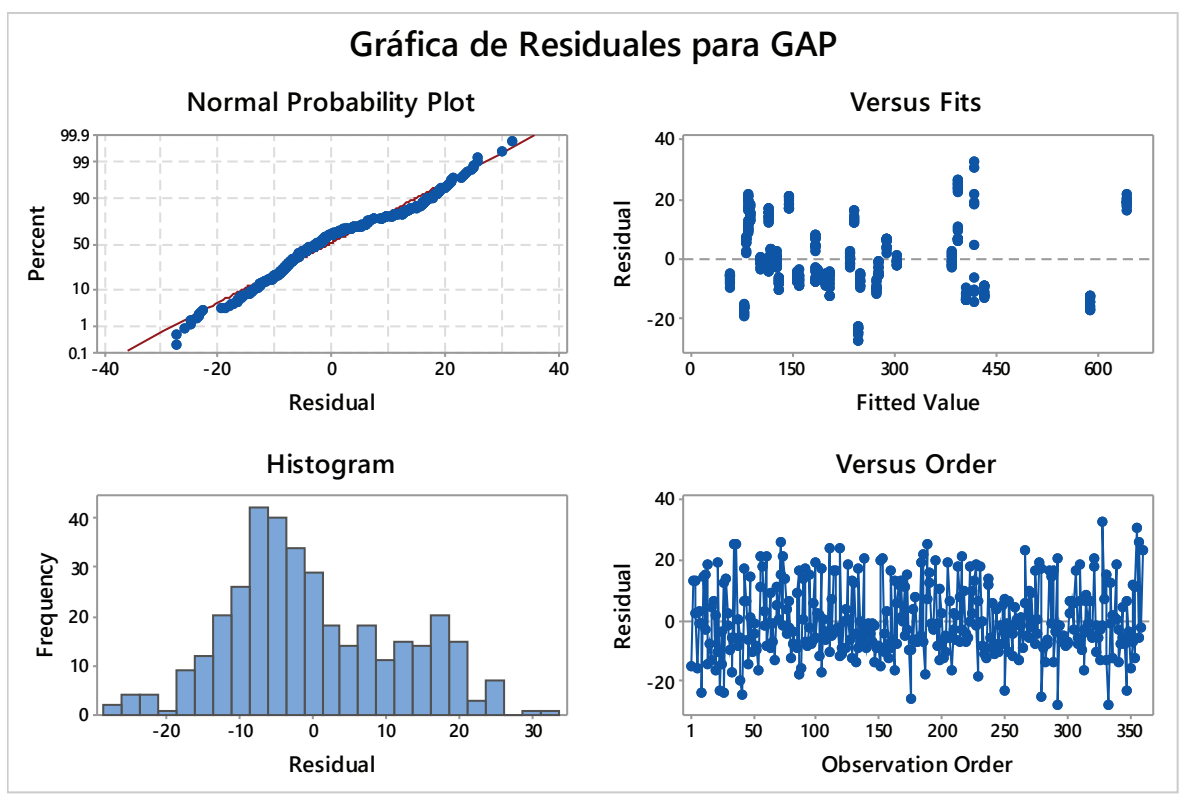

Figura 3. Análisis de residuales para gap de dualidad

Tabla 2. ANOVA para gap de dualidad

\begin{tabular}{cccccccc}
\hline Factores & g1 & $\begin{array}{c}\text { Suma } \\
\text { Cuadrados }\end{array}$ & $\begin{array}{c}\text { Media } \\
\text { Cuadrado }\end{array}$ & F & p-valor & (\%) Contri & $\begin{array}{c}\text { (\%) Contri } \\
\text { Acum. }\end{array}$ \\
\hline F2 & 2 & 3744.16 & 1872.08 & 19.13 & 0.0000 & 21.15 & 21.15 \\
F1 & 2 & 2336.61 & 1168.31 & 11.94 & 0.0000 & 13.20 & 34.34 \\
F1*F2 & 4 & 1290.83 & 322.71 & 3.3 & 0.0140 & 7.29 & 41.64 \\
F4 & 1 & 495.37 & 495.37 & 5.06 & 0.0270 & 2.80 & 44.43 \\
F2*F3 & 2 & 475.18 & 237.59 & 2.43 & 0.0940 & 2.68 & 47.12 \\
F3 & 1 & 282.65 & 282.65 & 2.89 & 0.0930 & 1.60 & 48.71 \\
F2*F4 & 2 & 280.78 & 140.39 & 1.43 & 0.2440 & 1.59 & 50.30 \\
F3*F4 & 1 & 98.87 & 98.87 & 1.01 & 0.3180 & 0.56 & 50.86 \\
F1*F4 & 2 & 59.35 & 29.67 & 0.3 & 0.7390 & 0.34 & 51.19 \\
F1*F3 & 2 & 30.48 & 15.24 & 0.16 & 0.8560 & 0.17 & 51.36 \\
Error & 340 & 8610.93 & 25.32 & & & 48.63 & 100.00 \\
Total & 359 & 17705.22 & & & & & \\
\hline$S=9.891 ~ R^{2}=51.37 \%$ & $R_{\text {(ajustado) }}^{2}$ & $=40.86 \%$ & & & & &
\end{tabular}


Smirnov ( $p$-valor $=0.010)$ indica que el supuesto de normalidad es significativo a un nivel $\alpha=0.010$; el estadístico Durbin-Watson $=2.3987$ indica que se cumple el su- puesto de independencia y la prueba de Levene ( $p$-valor $=0.153$ ) para igualdad de varianza muestra que se cumple el supuesto de homocedasticidad.

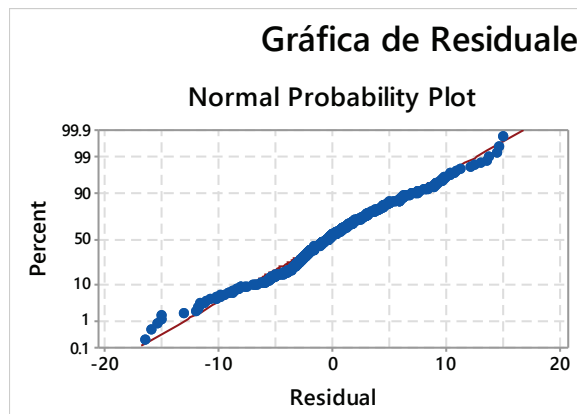

\section{para Tiempo de Ocio}
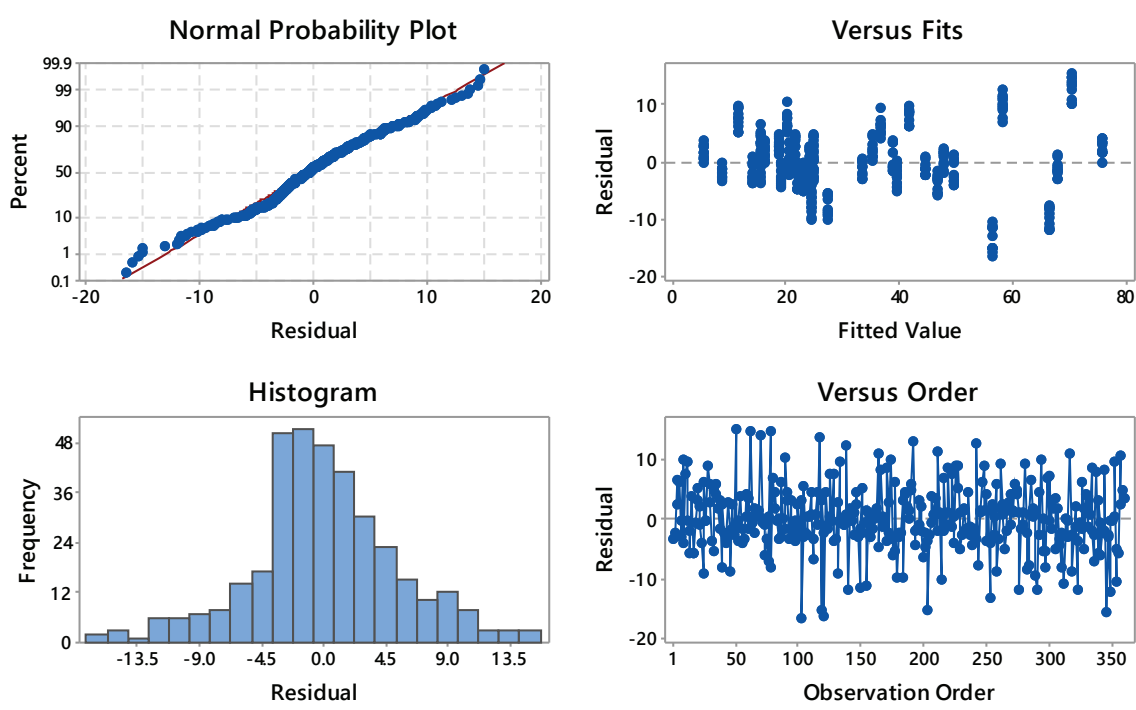

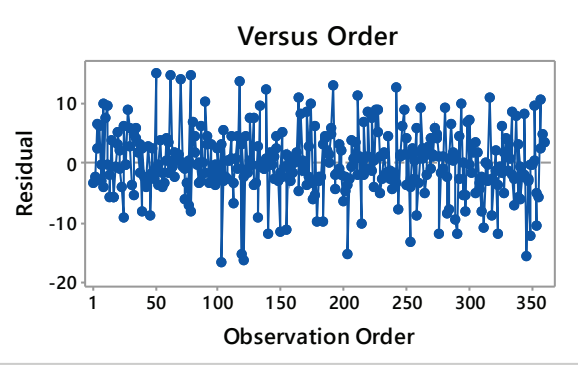

Figura 4. Análisis de residuales para tiempo de ocio

Tabla 3. ANOVA para tiempo de ocio total

\begin{tabular}{cccccccc}
\hline Factores & gl & $\begin{array}{c}\text { Suma } \\
\text { Cuadrados }\end{array}$ & $\begin{array}{c}\text { Media } \\
\text { Cuadrado }\end{array}$ & F & p-valor & (\%) Contri & $\begin{array}{c}\text { (\%) Contri } \\
\text { Acum. }\end{array}$ \\
\hline F4 & 1 & 28255.00 & 28255.00 & 51.26 & 0.0000 & 26.61 & 26.61 \\
F1 & 2 & 15973.50 & 7986.70 & 14.49 & 0.0000 & 15.05 & 41.66 \\
F1*F4 & 2 & 5375.50 & 2687.70 & 4.88 & 0.0100 & 5.06 & 46.72 \\
F2 & 2 & 4118.20 & 2059.10 & 3.74 & 0.0280 & 3.88 & 50.60 \\
F1*F3 & 2 & 898.50 & 449.20 & 0.81 & 0.4460 & 0.85 & 51.45 \\
F3*F4 & 1 & 882.50 & 882.50 & 1.60 & 0.2090 & 0.83 & 52.28 \\
F2*F4 & 2 & 800.50 & 400.20 & 0.73 & 0.4870 & 0.75 & 53.04 \\
F1 ${ }^{*}$ F2 & 4 & 757.10 & 189.30 & 0.34 & 0.8480 & 0.71 & 53.75 \\
F2*F3 & 2 & 492.60 & 246.30 & 0.45 & 0.6410 & 0.46 & 54.21 \\
F3 & 1 & 99.70 & 99.70 & 0.18 & 0.6720 & 0.09 & 54.31 \\
Error & 340 & 48509.10 & 142.67 & & & 45.69 & 100.00 \\
\hline Total & 359 & 106162.10 & & & & & \\
\hline $\mathrm{S}=23.478$ & $\mathrm{R}^{2}=54.31 \%$ & $\mathrm{R}_{\text {(ajustado) }}^{2}=44.44 \%$ & & & & &
\end{tabular}

Los resultados del ANOVA para el tiempo de ocio total (tabla 3) indican que tres de los cuatro factores son estadísticamente significativos. Tanto la capacidad de servicio de la terminal (F1) como la configuración de los arribos (F4) y la interacción entre ellos, son los principales responsables del tiempo de ocio total. La demanda (F2) también es un factor significativo al 0.05. En su conjunto estos factores explican $50.60 \%$ de la varianza total del tiempo de ocio. No existe suficiente evidencia estadística para confirmar que la variabilidad del tiempo de servicio (F3) y su interacción con los demás factores sean significativos. El valor del $R^{2}$ ajustado $=44.44 \%$, implica un ajuste medio del modelo. Se hace notar que cuando la variable dependiente es el tiempo total (tiempo de ocio total + tiempo total de Servicio), los mismos factores (F1, F2, F4) son significativos, así como la interacción de F1 con F2, y F2 con F4, en su conjunto explican $94.25 \%$ de la variabilidad del tiempo total; además 
de que en este caso el supuesto de normalidad es más evidente. El análisis de los residuales (figura 4) muestra que los supuestos del ANOVA se satisfacen: la prueba de normalidad de Kolgomorov-Smirnov ( $p$-valor=0.010) indica que el supuesto de normalidad es significativo a un nivel $\alpha=0.010$; el estadístico Durbin-Watson $=2.1351$ indica que se cumple el supuesto de independencia; y la prueba de Levene ( $p$-valor $=0.869)$ para igualdad de varianza muestra que se cumple el supuesto de homocedasticidad.

Se analizaron las matrices de variabilidad, error y de correlación parcial para evaluar el rendimiento del MANOVA. Para estimar la relación entre las variables de respuesta, se calcula la correlación parcial entre ambas, obteniendo un valor de 0.4824 . Por lo que la estructura de correlación es moderada, y por ello el uso del MANOVA es adecuado. Se hace uso de las pruebas de Wilks, Lawley-Hotelling y Pillai, para juzgar si existe evidencia significativa para los efectos del modelo. En la tabla 4, el $p$-valor para cada prueba muestra que los factores F1 (capacidad de servicio de la terminal), F2 (demanda), F4 (configuración de los arribos) y las interacciones entre $\mathrm{F} 1$ y $\mathrm{F} 4$ son característicos a un nivel de significancia $\alpha=0.05$; es decir, un cambio en los niveles de tales factores afectan las variables de respuesta de forma diferente. La tabla 4 muestra también la contribución relativa de cada factor a explicar cada variable de respuesta. Se utilizó el análisis Eigen para evaluar cómo difiere la media de las variables de respuesta entre los niveles de los diferentes términos del modelo. A partir de los resultados del análisis Eigen, es evidente que la media del gap de dualidad presenta mayor diferencia (variabilidad) entre los niveles de los siguientes factores significativos: capacidad de servicio de la ter- minal (F1) y demanda (F2). Sin embargo, la mayor diferencia entre las medias para el tiempo de ocio total se presenta en la configuración de los arribos (F4) y en la interacción de este factor con la capacidad de servicio de la terminal (F1).

Del análisis previo se concluye que:

a) El número de sitios de atraque disponibles en una terminal es un factor estadísticamente significativo para ambas variables de respuesta, y en la mayoría de los casos es la principal responsable de la variabilidad observada.

b) El número de buques que llegan a una terminal también es estadísticamente significativo para las dos variables de respuesta, pero este afecta principalmente la complejidad del DBAP.

c) La interacción del número de sitios de atraque con la variabilidad en la llegada de los buques es estadísticamente significativa, principalmente para el tiempo de ocio total.

d) Existe suficiente evidencia estadística para concluir que los mismos factores que afectan la productividad de los sitios de atraque, sirven para explicar la complejidad del problema de decisión asociado (el DBAP).

\section{DisCuSIÓN E IMPLICACIONES PRÁCTICAS}

Debido al incremento en el tráfico mundial de contenedores, la administración de operaciones en las terminales de contenedores se ha convertido en un elemento crucial para lograr niveles de eficiencia deseados. Una buena planificación de los sitios de atraque tiene un impacto positivo en ahorro de costos, como resultado de

Tabla 4. Resultados del MANOVA

\begin{tabular}{cccccccc}
\hline & \multicolumn{3}{c}{ Pruebas (p-valor) } & \multicolumn{3}{c}{ Contribución a la varianza (\%) } & \multicolumn{2}{c}{ Eigen-vectores } \\
\cline { 2 - 7 } Fuente & Wilk & $\begin{array}{c}\text { Lawley- } \\
\text { Hotelling }\end{array}$ & Pillai & $\begin{array}{c}\text { Tiempo de } \\
\text { Ocio Total }\end{array}$ & $\begin{array}{c}\text { Gap de } \\
\text { dualidad }\end{array}$ & $\begin{array}{c}\text { Tiempo de } \\
\text { Ocio Total }\end{array}$ & $\begin{array}{c}\text { Gap de } \\
\text { dualidad }\end{array}$ \\
\hline F1 & 0.000 & 0.000 & 0.000 & 15.05 & 13.20 & 0.003 & 0.006 \\
F2 & 0.000 & 0.000 & 0.000 & 3.88 & 21.15 & -0.001 & 0.011 \\
F3 & 0.233 & 0.233 & 0.233 & 0.09 & 1.60 & -0.001 & 0.012 \\
F4 & 0.000 & 0.000 & 0.000 & 26.61 & 2.80 & 0.005 & -0.001 \\
F1*F2 & 0.121 & 0.111 & 0.132 & 0.71 & 7.29 & -0.001 & 0.011 \\
F1*F3 & 0.555 & 0.556 & 0.553 & 0.85 & 0.17 & 0.005 & -0.008 \\
F1*F4 & 0.049 & 0.047 & 0.053 & 5.06 & 0.34 & 0.005 & -0.002 \\
F2*F3 & 0.286 & 0.287 & 0.286 & 0.46 & 2.68 & -0.003 & 0.011 \\
F2*F4 & 0.189 & 0.189 & 0.189 & 0.75 & 1.59 & -0.004 & 0.010 \\
F3*F4 & 0.400 & 0.400 & 0.400 & 0.83 & 0.56 & 0.003 & 0.005 \\
Error & & & & 45.69 & 48.63 & & \\
\hline
\end{tabular}


un uso eficiente de recursos (muelle, grúas, operadores, etcétera) y un incremento en los niveles de servicio a los clientes (líneas navieras). El que la variabilidad en el tiempo de servicio no sea un factor estadísticamente significativo para la productividad de los sitios de atraque, implica que una estrategia orientada a tratar de equilibrar la capacidad de servicio en los sitios de atraque, no mejorará la productividad. En este sentido, una mejor estrategia debería estar orientada a maximizar la flexibilidad de los sitios de atraque para ajustar su capacidad de servicio a la demanda de movimientos que requieran los buques.

La variabilidad en el tiempo de llegada de los buques presenta un impacto negativo sobre la productividad de los sitios de atraque. Cuando la llegada de los buques es homogénea (el tiempo de llegada entre buques presenta poca variabilidad), la productividad de los sitios de atraque se incrementan conforme disminuye el número de sitios de atraque disponibles. Del mismo modo, cuando la llegada de los buques es homogénea, la productividad de los sitios de atraque se incrementa conforme disminuye el número de buques a atender. Por otro lado, cuando la llegada de los buques es heterogénea (el tiempo de llegada entre buques presenta mayor variabilidad), la productividad de los sitios de atraque tiende a incrementarse cuando aumenta el número de buques a atender; sin embargo, el efecto resultante es una mayor variabilidad en los tiempos de ocio (figura 5). Esto se puede explicar de la siguiente manera: si grupos homogéneos de buques llegan en intervalos pequeños de tiempo, se facilita la programación de los servicios, a expensas de los tiempos de espera de los buques. Por lo que una estrategia adecuada para una terminal de contenedores con llegada de buques homogénea estaría orientada a reducir la congestión en los sitios de atraque (el cociente entre el número de buques y el número de sitios de atraque), mediante el aumento de sitios de atraque, o el incremento de recursos de operación en muelle (por ejemplo, grúas pórtico y vehículos internos) y patios (por ejemplo, grúas de patio y bloques de contenedores) y la implementación de mejores prácticas de operación (por ejemplo, pre-clasificación de contenedores para reducir el número de reacomodos).

\section{Conclusiones}

Este artículo examina el impacto que tienen algunos factores operacionales (por ejemplo, la variabilidad en los tiempos de servicio) y estratégicos (por ejemplo, el número de sitios de atraque) sobre la productividad de los sitios de atraque y la complejidad de su problema de optimización asociado. Como base para la experimentación, se utilizó la formulación propuesta por Hansen y Oguz (2003) para el problema dinámico de asignación de sitios de atraque, haciendo uso del IBM ILOG CPLEX Optimization Studio para el modelado y solución de las instancias. Los resultados de estudio computacional se analizaron a partir de un enfoque de diseño experimental basado en el análisis de varianza y el análisis multivariado de varianza, para probar simultáneamente si la productividad de los sitios de atraque y la complejidad del problema de optimización asociado se ven alterados por la manipulación de algunos factores. El análisis estadístico revela que el número de sitios de atraque, el número de buques y su variabilidad en los tiempos de llegada son estadísticamente significativos para la productividad de los sitios de atraque y la complejidad del problema de optimización asociado.

Cabe destacar que los resultados de esta investigación constituyen una primera aproximación al diseño y formulación de estrategias de operación eficientes para
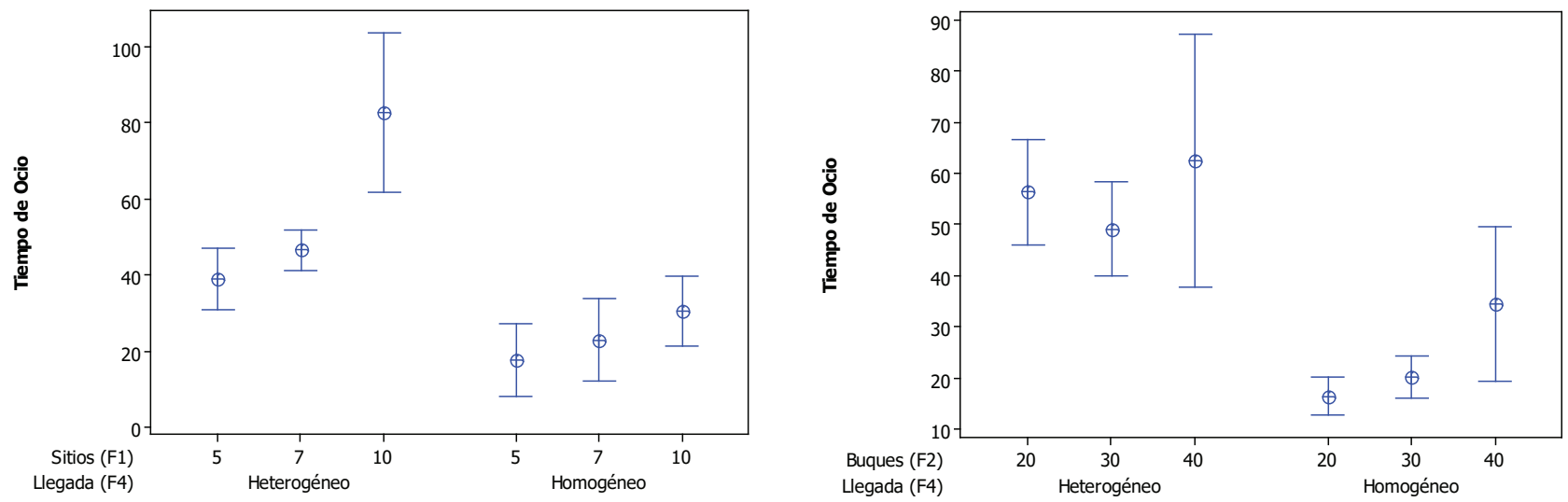

Figura 5. Impacto de los factores F1, F2 y F4 sobre el tiempo de ocio de los sitios de atraque 
la gestión de terminales de contenedores. Como trabajo futuro se plantea utilizar el mismo esquema metodológico en el estudio de otros problemas de decisión que integra varios problemas de optimización, por ejemplo el minimizar el ship turnarround time que integra varios problemas de optimización relacionados con la planificación naviera y de patio, tales como el stowage planning, quay crane assigment and schedulling, entre otros. Otra propuesta de trabajo futuro, es investigar el impacto que puede tener el incremento en el tamaño de los buques y la disminución en las frecuencias de llegada a las terminales sobre la eficiencia de una terminal.

\section{RefERENCIAS}

Bierwirth C. y Meisel F. A follow-up survey of berth allocation and quay crane scheduling problems in container terminals. European Journal of Operational Research, volumen 244 (número 3), agosto 2015: 675-689.

Bierwirth C. y Meisel F. A survey of berth allocation and quay crane scheduling problems in container terminals. European Journal of Operational Research, volumen 202 (número 3), mayo 2010: 615-627.

Böse J.W. Handbook of terminal planning, 1a ed., Berlin, SpringerVerlag, 2011.

Carlo H.J., Vis I.F., Roodbergen K.J. Seaside operations in container terminals: Literature overview, trends, and research directions. Flexible Services and Manufacturing Journal, volumen 27 (número 2-3), septiembre 2015: 224-262.

Carlo H.J., Vis I.F., Roodbergen K.J. Storage yard operations in container terminals: Literature overview, trends, and research directions. European Journal of Operational Research, volumen 235 (número 2), junio 2014: 412-430.

Comisión Económica para América Latina y el Caribe (CEPAL). Ranking 2014 de puertos de contenedores de América Latina y el Caribe, 2015 [en línea] [fecha de consulta: 30 de noviembre de 2015]. Disponible en: http://www.cepal.org/cgi-bin/ getProd.asp?xml=/perfil/noticias/noticias/9/53129/P53129. xml\&xsl=/perfil/tpl/p1f.xsl\&base=/perfil/tpl/top-bottom.xsl

Cordeau J.F., Laporte G., Legato P., Moccia L. Models and tabu search heuristics for the berth-allocation problem. Transportation Science, volumen 39 (número 4), noviembre 2005: 526-538.

Derpich I. y Vera R. Improving the efficiency of the Branch and Bound algorithm for integer programming based on flatness information. European Journal of Operational Research, volumen 174 (número 1), octubre 2006: 92-101.

Du Y., Chen Q., Lam, J.S.L., Xu Y., Cao J.X. Modeling the impacts of tides and the virtual arrival policy in berth allocation. Transportation Science, voumen 49 (número 4), noviembre 2015: 939-956.

Frojan P., Correcher J.F., Alvarez-Valdes R., Koulouris G., Tamarit J.M. The continuous Berth Allocation Problem in a container terminal with multiple quays. Expert Systems with Applications, volumen 42 (número 21), noviembre 2015: 7356-7366.

Garey M.R. y Johnson, D.S. Computers and intractability: a guide to the theory of NP-completeness, 1a ed., San Francisco, L.A., Freeman, 1979.

Giallombardo G., Moccia L., Salani M., Vacca I. Modeling and solving the tactical berth allocation problem. Transportation Research Part B: Methodological, volumen 44 (número 2), febrero 2010: 232-245.

Günther H.O. y Kim K.H (Eds.). Container terminals and automated transport systems: logistics control issues and quantitative decision support, 1a ed., Heidelberg, Springer-Verlag, 2005.

Hansen P., Oguz C., Mladenovic N. Variable neighborhood search for minimum cost berth allocation. European Journal of Operational Research, volumen 191 (número 3), diciembre 2008: 636-649.

Hansen P. y Oguz C. A note on formulations of the static and dynamic berth allocation problems, Les Cahiers du GERAD, HEC Montréal, 2003, 17 p.

$\mathrm{Hu}$ Z.H. Heuristics for solving continuous berth allocation problem considering periodic balancing utilization of cranes. Computers and Industrial Engineering, volumen 85 (número 1), julio 2015: 216-226.

Imai A., Nagaiwa K., Tat C.W. Efficient planning of berth allocation container terminals in Asia. Journal of Advanced Transportation, volumen 31 (número 1), 1997: 75-94.

Imai A., Nishimura E., Papadimitriou S. The dynamic berth allocation problem for a container port. Transportation Research Part B: Methodological, volumen 35 (número 4), mayo 2001: 401-417.

Iris Ç., Pacino D., Ropke S., Larsen A. Integrated berth allocation and quay crane assignment problem: set partitioning models and computational results. Transportation Research Part E: Logistics and Transportation Review, volumen 81 (número 1), septiembre 2015: 75-97.

Kim K.H. y Günther H.O. Container terminals and cargo systems: design, operations management, and logistics control issues, 1a ed., Berlin, Springer-Verlag, 2007.

Lim A. The berth planning problem. Operations Research Letters, volumen 22 (número 2-3), marzo 1998: 105-110.

Macharis C. y Bontekoning Y.M. Opportunities for OR in intermodal freight transport research: a review. European Journal of Operational Research, volumen 153 (número 2), marzo 2004: 400-416.

Mar-Ortiz J., Gracia M.D., González-Ramírez R.G. A Framework for Improving Logistics Operations at Container Terminals, en Handbook of Research on Managerial Strategies for Achieving Optimal Performance in Industrial Processes, $1^{\text {st }}$ ed., Hershey PA, USA, IGI Global, 2016, pp. 1-23.

Meisel F. y Bierwirth C. A framework for integrated berth allocation and crane operations planning in seaport container terminals. Transportation Science, volumen 47 (número 2), mayo 2013: 131-147. 
Meisel F. y Bierwirth C. Heuristics for the integration of crane productivity in the berth allocation problem. Transportation Research Part E: Logistics and Transportation Review, volumen 45 (número 1), enero 2009: 196-209.

Nishimura E., Imai A., Papadimitriou S. Berth allocation planning in the public berth system by genetic algorithms. European Journal of Operational Research, volumen 131 (número 2), junio 2001: 282-292.

Park K.T. y Kim K.H. Berth scheduling for container terminals by using sub-gradient optimization technique. Journal of the Operational Research Society, volumen 53 (número 9), septiembre 2002: 1054-1062.

Park Y.M. y Kim K.H. A scheduling method for berth and quay crane. OR Spectrum, volumen 25 (número 1), febrero 2003: 1-23.

Secretaría de Comunicaciones y Transporte (SCT). Estadísticas de Transporte de America del Norte - Principales puertos según movimientos de TEU [en línea], DT 11-2, NATS-SCT, 2015. [fecha de consulta: 30 de noviembre de 2015]. Disponible en: http://nats.sct.gob.mx.

Steenken D., Voß S., Stahlbock R. Container terminal operation and operations research- a classification and literature review. OR Spectrum, volumen 26 (número 1), enero 2004: 3-49.

Tao Y. y Lee C.Y. Joint planning of berth and yard allocation in transshipment terminals using multi-cluster stacking strategy. Transportation Research Part E: Logistics and Transportation Review, volumen 83 (número 34-50), noviembre 2015: 34-50.
United Nations Conference on Trade and Development (UNCTAD). Review of Maritime Transport 2015 [en línea], United Nations, New York and Ginebra, 2015. [fecha de consulta: 30 de noviembre de 2015]. Disponible en: http://unctad. org/en/PublicationsLibrary/rmt2015_en.pdf

Vis I.F.A. y de Koster R. Transshipment of containers at a container terminal: an overview. European Journal of Operational Research, volumen 147 (número 1), mayo 2003: 1-16.

Zhen L. Tactical berth allocation under uncertainty. European Journal of Operational Research, volumen 247 (número 3), diciembre 2015: 928-944.

\section{Citación sugerida:}

\section{Citación estilo Chicago}

Mar-Ortiz, Julio, María D. Gracia. Un analisis de los factores que afectan la productividad de los sitios de atraque en una terminal de contenedores. Ingeniería Investigación y Tecnología, XVIII, 02 (2017): 169-181.

\section{Citación estilo ISO 690}

Mar-Ortiz J., Gracia M.D. Un analisis de los factores que afectan la productividad de los sitios de atraque en una terminal de contenedores. Ingeniería Investigación y Tecnología, volumen XVIII (número 2), abril-junio 2017: 169-181.

\section{SemblanZas de los Autores}

Julio Mar-Ortiz. Es profesor de tiempo completo en la Facultad de Ingeniería de la Universidad Autónoma de Tamaulipas, México. Doctor en ingeniería industrial con especialidad en investigación de operaciones. Líder del cuerpo académico en productividad y optimización y miembro del Sistema Nacional de Investigadores. Sus investigaciones se han publicado en revistas como: el Journal of the Operational Research Society, Journal of Heuristics, The International Journal of Advanced Manufacturing Technology.

María D. Gracia. Es profesor de tiempo completo en la Facultad de Ingeniería de la Universidad Autónoma de Tamaulipas, México. Doctora en ciencias de la ingeniería con especialidad en gestión de operaciones. Sus áreas de interés son la gestión de cadenas de suministro y la formulación de estrategias. Sus investigaciones se han publicado en revistas científicas como: el NETNOMICS: Economic Research and Electronic Networking, e Ingeniería, Investigación y Tecnología. 